\title{
Practical Adaptive Oblivious Transfer from Simple Assumptions
}

\author{
Matthew Green ${ }^{\star}$ and Susan Hohenberger ${ }^{\star \star}$ \\ Johns Hopkins University \\ \{mgreen, susan\}@cs.jhu.edu
}

\begin{abstract}
In an adaptive oblivious transfer (OT) protocol, a sender commits to a database of messages and then repeatedly interacts with a receiver in such a way that the receiver obtains one message per interaction of his choice (and nothing more) while the sender learns nothing about any of the choices. Recently, there has been significant effort to design practical adaptive OT schemes and to use these protocols as a building block for larger database applications. To be well suited for these applications, the underlying OT protocol should: (1) support an efficient initialization phase where one commitment can support an arbitrary number of receivers who are guaranteed of having the same view of the database, (2) execute transfers in time independent of the size of the database, and (3) satisfy a strong notion of security under a simple assumption in the standard model.

We present the first adaptive OT protocol simultaneously satisfying these requirements. The sole complexity assumption required is that given $\left(g, g^{a}, g^{b}, g^{c}, Q\right)$, where $g$ generates a bilinear group of prime order $p$ and $a, b, c$ are selected randomly from $\mathbb{Z}_{p}$, it is hard to decide if $Q=g^{a b c}$. All prior protocols in the standard model either do not meet our efficiency requirements or require dynamic " $q$-based" assumptions.

Our construction makes an important change to the established "assisted decryption" technique for designing adaptive OT. As in prior works, the sender commits to a database of $n$ messages by publishing an encryption of each message and a signature on each encryption. Then, each transfer phase can be executed in time independent of $n$ as the receiver blinds one of the encryptions and proves knowledge of the blinding factors and a signature on this encryption, after which the sender helps the receiver decrypt the chosen ciphertext. One of the main obstacles to designing an adaptive OT scheme from a simple assumption is realizing a suitable signature for this purpose (i.e., enabling signatures on group elements in a manner that later allows for efficient proofs.) We make the observation that a secure signature scheme is not necessary for this paradigm, provided that signatures can only be forged in certain ways.
\end{abstract}

* Supported by NSF CNS-1010928. This publication was also made possible by Grant Number HHS 90TR0003/01. Its contents are solely the responsibility of the authors and do not necessarily represent the official views of the HHS.

** Supported by NSF CAREER CNS-1053886, a Microsoft New Faculty Fellowship and a Google Research Award. 
We then show how to efficiently integrate an insecure signature into a secure adaptive OT construction.

\section{Introduction}

Oblivious transfer OT 35139] is a two-party protocol, where a Sender with messages $M_{1}, \ldots, M_{N}$ and a Receiver with indices $\sigma_{1}, \ldots, \sigma_{k} \in[1, N]$ interact in such a way that at the end the Receiver obtains $M_{\sigma_{1}}, \ldots, M_{\sigma_{k}}$ without learning anything about the other messages and the Sender does not learn anything about the choices $\sigma_{1}, \ldots, \sigma_{k}$. In the adaptive OT setting [33, the Receiver may obtain $M_{\sigma_{i-1}}$ before deciding on $\sigma_{i}[33$.

Our Goals. Adaptive OT is an interesting primitive. Like non-adaptive OT, it is a key building block for secure multi-party computation 40,1928. More practically, it captures the way an oblivious medical, financial or patent database would be accessed. Recently, there has been a focus on designing practical, privacypreserving databases with access controls [15] or pricing mechanisms [36] based on adaptive OT. Unfortunately, researchers trying to design more-complex systems on top of current adaptive OT protocols do not have any ideal choices. For a database with $N$ messages supporting $U$ Receivers with security parameter $\lambda$, such a protocol must be:

1. Extremely efficient, even when $N$, the database size, is large. In particular, the cost to transfer one message to one Receiver should depend only on the security parameter and not on $N$. I.e., a Receiver should not have to do work proportional to the size of the database to download one file. (This rules out a number of naive approaches as discussed below.)

2. Furthermore, since few databases serve only one user, it should be possible to extend the protocol to the case where there are many Receivers, each of whom receives a consistent view of the database. In particular, the ideal situation, which we achieve in this work, is to have a non-interactive initialization phase, where the Sender can do $O(\lambda N)$ work to form a commitment that can then be used for an arbitrary number of receivers. Several prior works (e.g., 10222736 ) support a relatively efficient initialization phase with $O(\lambda(N+U))$ total work. By adding a CRS and making some modifications, this can likely be reduced to $O(\lambda N)$ (although the complexity assumptions will still be an issue.) What one wishes to avoid, however, is an initialization phase that requires $O(\lambda N U)$ total work. I.e., the sender should not have to set up a unique database containing all of its files for each of its users. (This also rules out some basic approaches.)

3. Finally, since this protocol is designed to be a building block of larger applications, it is critical that it be a solid one. In particular, it should satisfy a strong notion of security (i.e., full-simulatability or UC) under a mild complexity assumption in the standard model. Unfortunately, while 
sufficiently practical protocols exist, they either require random oracles

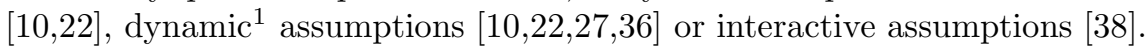

Thus, a new construction based on new techniques is needed.

From Non-Adaptive to Adaptive OT for Single Receivers. Since it is known how to build non-adaptive OT protocols based on simple assumptions [213234] such as Decisional Diffie-Hellman and Quadratic Residuosity, it is natural to ask why constructing adaptive protocols has proven so difficult. Given any fully-simulatable 1-out-of- $N$ non-adaptive OT protocol, one can build a fully-simulatable $k$-out-of$N$ adaptive OT protocol for a single Receiver by sequentially executing $k$ instances of the non-adaptive protocol and, before each execution, having the sender prove in zero-knowledge that the sequence of $N$ messages used in execution $i$ is the same as the sequence of $N$ messages used in execution $i-1$ [10. Unfortunately, for security parameter $\lambda$, this protocol requires a total of $O(N k \lambda)$ work to transfer $k$ messages for (only) one Receiver and is thus impractical for any application involving large databases.

Thus, when Camenisch, Neven and shelat [10] began to reinvestigate this problem in 2007, they stressed that the real challenge was to build an OT scheme where the sender makes an initial commitment to the database (which is assumed to be broadcast to all receivers), and then the two parties only exchange a constant number of group elements per transfer.

Our Contributions. We present an efficient, adaptive oblivious transfer protocol which is fully-simulatable under a simple, static assumption. The sole complexity assumption required is that given $\left(g, g^{a}, g^{b}, g^{c}, Q\right)$, where $g$ generates a bilinear group of prime order $p$ and $a, b, c$ are selected randomly from $\mathbb{Z}_{p}$, it is hard to decide if $Q=g^{a b c}$. This assumption called Decisional 3-Party Diffie-Hellman has been used in prior works 31525 . Our protocol is practical, although more costly than the very efficient Camenisch et al. protocol [10] by a constant factor. The database commitment in our scheme requires roughly $(9+7 N)$ group elements, whereas the commitment in [10] required roughly $(3+2 N)$ group elements. By including the mild Decision Linear assumption [4, we can efficiently make this initialization phase non-interactive as we discuss in Section 3.

Our construction introduces a twist on the assisted decryption approach to OT design, where the underlying signatures need not be existentially unforgeable provided that certain forgeries are not permitted. As we discuss, these techniques may be useful in simplifying the complexity assumptions in schemes beyond OT such as $F$-signatures and anonymous credentials [1.

${ }^{1}$ These are also called parametric or $q$-based assumptions. An example is $q$-Strong Diffie-Hellman 3 ] $\left(q\right.$-SDH): given $\left(g, g^{x}, g^{x^{2}}, g^{x^{3}}, \ldots, g^{x^{q}}\right)$, where $g$ generates a group of prime order $p$ and $x$ is a random value in $\mathbb{Z}_{p}$, it is hard to compute $\left(g^{1 /(x+c)}, c\right)$ for any $c \in \mathbb{Z}_{p}^{*}$. Typically, when $q$-SDH is used as the foundation of an adaptive OT scheme, $q$ must dynamically adjust to the number of files in the database. Thus, the assumption required actually changes based on how the protocol is used. 


\begin{tabular}{|c|c|c|c|c|}
\hline Protocol & $\begin{array}{c}\text { Initialization } \\
\text { Cost }\end{array}$ & $\begin{array}{c}\text { Transfer } \\
\text { Cost }\end{array}$ & Assumption & $\begin{array}{l}\text { Security } \\
\text { Defn }\end{array}$ \\
\hline Folklore & & $O(\lambda N)$ & general assumptions & Full Sim \\
\hline KN 29] & $O(\lambda(N+U))$ & $O(\lambda N)$ & Decisional $n$th Residuosity/DDH & Full Sim \\
\hline NP 33 & -1 & $O(\lambda \lg (N))$ & $\mathrm{DDH}+\mathrm{OT}_{1}^{2}$ & Half Sim \\
\hline KNP [30] & $O(\lambda N U)$ & $O(\lambda)$ & $\overline{\mathrm{DDH}}$ & Full Sim* \\
\hline CNS [10] & $O(\lambda(N+U))$ & $O(\lambda)$ & $q$-Power DDH $+q$-Strong DH & Full Sim \\
\hline GH 22 & $O(\lambda(N+U))$ & $O(\lambda)$ & Decision Linear $+q$-Hidden LRSW & $\mathrm{UC}$ \\
\hline JL [27] & $O(\lambda(N+U))$ & $O(\lambda)$ & Comp. Dec. Residuosity $+q$-DDHI & Full Sim \\
\hline RKP 36 & $O(\lambda(N+U))$ & $O(\lambda)$ & $\mathrm{DLIN}+q$-Hidden $\mathrm{SDH}+q$-TDH & $\mathrm{UC}$ \\
\hline$\$ 2.1$ & $O(\lambda(N+U))$ & $O(\lambda)$ & Decision 3-Party DH & Full Sim \\
\hline$\$ 3$ & $O(\lambda N)$ & $O(\lambda)$ & Decision 3-Party DH + DLIN & Full Sim \\
\hline
\end{tabular}

Fig. 1. Survey of adaptive $k$-out-of- $N$ Oblivious Transfer protocols secure in the standard model. Let $\lambda$ be the security parameter, $N$ the size of the database and $U$ the number of receivers. The horizontal lines separate the schemes into efficiency categories, which improve as one scans down the table. While the least efficient categories can be realized using assumptions such as DDH, all prior attempts to achieve practicality have required a dynamic $q$-based complexity assumption. A $*$ denotes the construction meets a strictly weaker notion than the standard used in the other works.

Intuition behind our $\mathrm{OT}_{k \times 1}^{N}$ Construction. As with most previous $\mathrm{OT}_{k \times 1}^{N}$ constructions, our construction uses a technique known as assisted decryption. For $i=1$ to $N$, the Sender commits to his database by encrypting each message as $C_{i}=\operatorname{Enc}\left(M_{i}\right)$, and publishes a public key and ciphertexts $\left(p k, C_{1}, \ldots, C_{N}\right)$. The Receiver then checks that each ciphertext is well-formed. To obtain a message, the Sender and Receiver engage in a blind decryption protocol, i.e., an interactive protocol in which the Sender does not view the ciphertext he decrypts, but where the Receiver is convinced that decryption was done correctly.

The difficulty here is to prevent the Receiver from abusing the decryption protocol, e.g., by requesting decryptions of ciphertexts which were either not produced by the Sender or have been mauled. The folklore solution is to have the Receiver provide a proof that his request corresponds to $C_{1} \vee C_{2} \vee \ldots \vee C_{N}$. Of course, the cost of each transfer is now dependent on the total database size and thus this solution is no (asymptotically) better than the trivial solution mentioned above.

In Eurocrypt 2007, Camenisch, Neven and shelat [10] were the first to propose a method for executing "assisted decryption" efficiently. The sender signed each ciphertext value. The receiver was required to prove knowledge of a corresponding signature before the sender would assist him in decrypting a ciphertext. This clever approach reduced the $O(N \lambda)$ work per transfer required above, to only $O(\lambda)$ work, where $\lambda$ is a security parameter.

More specifically, Camenisch, Neven and shelat [10] used a deterministic encryption scheme and a signature with a particular structure: for $p k=\left(g, g^{x}, H=\right.$ $e(g, h))$ and $s k=h$, let $C_{i}=\left(g^{\frac{1}{x+i}}, M_{i} \cdot e(g, h)^{\frac{1}{x+i}}\right)$. Recall that $g^{1 /(x+i)}$ is a weak Boneh-Boyen signature [3] on $i$ under $g^{x}$, and here only a polynomial 
number of "messages" (1 to $N$ ) are signed. While this scheme supports an elegant and efficient blind decryption protocol, it also requires strong $q$-based assumptions for both the indistinguishability of the ciphertexts as well as the unforgeability of the weak Boneh-Boyen signature. It is based on the $q$-Strong Diffie-Hellman and the $q$-Power Decisional Diffie-Hellman assumptions. The latter assumption states that given $\left(g, g^{x}, g^{x^{2}}, \ldots, g^{x^{q}}, H\right)$, where $g \in \mathbb{G}$ and $H \in$ $\mathbb{G}_{T}$, it is hard to distinguish the vector of elements $\left(H^{x}, H^{x^{2}}, \ldots, H^{x^{q}}\right)$ from a vector of random elements in $\mathbb{G}_{T}$. In essence, the rigid structure of this (and all prior) constructions appear to require a similarly structured complexity assumption, which grows with the database size.

To move past this, we will "loosen" the structure of the ciphertext and signature enough to break the dependence on a structured assumption, but not so much as to ruin our ability to perform efficient proofs. Finding this balance proved highly non-trivial.

We now turn to how our construction works. We will encrypt using the BonehBoyen IBE [2], which has a public key $p k=\left(g, g_{1}=g^{a}, g_{2}, h\right)$ and encrypts $M$ as $\left(g^{r},\left(g_{1}^{i} h\right)^{r}, e\left(g_{1}, g_{2}\right)^{r} M\right)$ for identity $i$ and randomness $r \in \mathbb{Z}_{p}$. Then we will sign $r$. To do this, we need a standard model signature scheme from a simple assumption (which is itself somewhat rare.) We choose the stateful signatures of Hohenberger-Waters [26], which has a public key $p k=\left(g, g^{b}, u, v, d, w, z, h\right)$ and signs $M$ as $\left(\sigma_{1}, \sigma_{2}, s, i\right)$ for state $i$ and randomness $s, t \in \mathbb{Z}_{p}$, where $\sigma_{1}=$ $g^{t}, \sigma_{2}=\left(u^{M} v^{s} d\right)^{b}\left(w^{\lceil\lg (i)\rceil} z^{i} h\right)^{t}$.

Attempt 1. Now, consider the construction obtained by combining the BB IBE, secure under Decisional Bilinear Diffie-Hellman, with the HW signature, secure under the Computational Diffie-Hellman assumption. Here we will encrypt the $i$ th message using identity $i$ (in the BB IBE) and state $i$ (in the HW signature), with an extra $u^{r}$ term to allow the Receiver to verify well-formedness:

$$
g^{r}, \quad\left(g_{1}^{i} h\right)^{r}, \quad e\left(g_{1}, g_{2}\right)^{r} M, \quad g^{t}, \quad\left(u^{r} v^{s} d\right)^{b}\left(w^{\lceil\lg (i)\rceil} z^{i} h\right)^{t}, \quad u^{r}, \quad s
$$

The Receiver can verify the well-formedness of the $i$ th ciphertext $\left(c_{1}, \ldots, c_{7}\right)$ by checking that $e\left(\left(g_{1}^{i} h\right), c_{1}\right)=e\left(g, c_{2}\right), e\left(g, c_{6}\right)=e\left(c_{1}, u\right)$ and

$$
e\left(g, c_{5}\right)=e\left(c_{6} v^{c_{7}} d, g^{b}\right) e\left(w^{\lceil\lg (i)\rceil} z^{i} h, c_{4}\right) .
$$

It is important that the Receiver can verify the well-formedness of the ciphertextsignature pair, so that the simulator can properly extract the messages from a cheating Sender during the proof of security. It is a nice additional feature that our verification is public and non-interactive.

Attempt 2. However, the above construction still has a lot of problems. Recall that we want the Receiver to ask for a blind decryption of a given ciphertext by (somehow) sending in blinded portions of the ciphertext, proving that these portions are linked to $r$ and proving that he knows a signature on $r$. Unfortunately, efficiently proving knowledge of the HW signature is problematic due to the $\lceil\lg (i)\rceil$ exponent. We could do this using a range proof [13967], however, this 
would require that we introduce stronger assumptions such as Strong RSA or $q$-Strong Diffie-Hellman. We could instead do a bit-by-bit proof, but this would severely hurt our efficiency. Instead, our solution is to drop this term entirely from the HW signature to obtain the ciphertext:

$$
g^{r}, \quad\left(g_{1}^{i} h\right)^{r}, \quad e\left(g_{1}, g_{2}\right)^{r} M, \quad g^{t}, \quad\left(u^{r} v^{s} d\right)^{b}\left(z^{i} h\right)^{t}, \quad u^{r}, \quad s
$$

One major issue is that dropping this term breaks the unforgeability of the signature scheme. Indeed, it is now possible for anyone to efficiently compute a signature on any index over a certain polynomial threshold as set in the proof of security. However, we specifically chose to encrypt with the Boneh-Boyen IBE for this purpose. We will set our parameters so that an adversary is free to forge signatures with states of $N+1$ and higher, where $N$ is the size of our database. The key idea is that asking for decryptions on different identities will not help a malicious Receiver obtain information about the database messages; indeed, we could even hand him the secret key for those identities. This makes our proof much more efficient, however, there is still a large problem.

Attempt 3. To argue, in the proof of security, that no malicious Receiver can forge signatures on a state $i \in[1, N]$, we must extract this signature and its forgery message from the proof of knowledge. However, we cannot extract the "message" $r$ from a cheating Receiver, because an honest Receiver will not know the randomness used in the ciphertexts created by the Sender. The most we can ask a Receiver to prove knowledge of is the signature on $r$ comprised of $\left(c_{4}, c_{5}, c_{6}, c_{7}\right)$ and the value $g^{r}$. Thus, we cannot extract from the Receiver a valid forgery of the HW signatures.

Moreover, we need a stronger security guarantee than HW signatures gave us (i.e., existential unforgeability under adaptive chosen message attack [20].) We need that: it is not only the case that an adversary cannot produce a pair $(m, \sigma)$ for a new $m$; now the adversary cannot even produce the pair $\left(g^{m}, \sigma\right)$ for a new $m$, where $\sigma$ is a signature on $m$. Do such powerful signatures exist?

Indeed, this security notion was formalized as $F$-signatures by Belenkiy, Chase, Kohlweiss and Lysyanskaya [1], where they also required $q$-based complexity assumptions for their construction. Fortunately, we are able to show that the HW signatures (and our mangled version of them without the $w^{\lceil\lg (i)\rceil}$ term) remain $F$-unforgeable for $F(m)=g^{m}$ under a simple static assumption. (See [26] or the full version of this work [23] for the full details on HW; the mangled version is proven as part of the OT system in Section 2.2.) We tie both this version of the signature scheme and the Boneh-Boyen IBE together under a single assumption: given $\left(g, g^{a}, g^{b}, g^{c}\right)$, it is hard to decide if $Q=g^{a b c}$.

Comparison to Prior Work. Let us briefly compare our approach to prior works; see Figure 1 for more. As we mention above, Camenisch, Neven and shelat [10] gave the first efficient, fully-simulatable construction for adaptive (and non-adaptive) OT. It is secure in the standard model under the $q$-Strong Diffie-Hellman and the $q$-Power Decisional Diffie-Hellman assumptions. 
They also provided a scheme in the random oracle model from unique blind signatures.

Green and Hohenberger 21 provided an adaptive OT construction in the random oracle model based on the Decisional Bilinear Diffie-Hellman assumption, namely, that given $\left(g, g^{a}, g^{b}, g^{c}, Q\right)$, it is hard to decide if $Q=e(g, g)^{a b c}$. In their construction, the Sender encrypted each message $i$ under identity $i$ using a IBE system. Then they provided a blind key extraction protocol, where the Receiver could blindly obtain a secret key for any identity of her choice.

In the assisted decryption setting, Green and Hohenberger 22 took an approach similar to [10] to achieve UC security. It was based on the Decision Linear and $q$-Hidden LRSW assumptions, in the asymmetric setting. The latter assumption implies that DDH must hold in both $\mathbb{G}_{1}$ and $\mathbb{G}_{2}$.

Jarecki and Liu 27 took an alternative view: for $p k=g^{x}$, let $C_{i}=M_{i}$. $g^{1 /(x+i)}$. Recall that $g^{1 /(x+i)}$ is also the Dodis-Yampolskiy pseudorandom function on input $i$ [18. This essentially simplifies the Camenisch et al. construction and allows a fully-simulatable scheme based on the Composite Decisional Residuosity and $q$-Decisional Diffie-Hellman Inversion assumptions. The blind decryption protocol involves obliviously evaluating the PRF on input $i$, which requires some costly zero knowledge proofs. However, this protocol is interesting as the only efficient and fully-simulatable protocol that does not require bilinear groups.

Rial, Kohlweiss and Preneel [36] presented a priced version of UC-secure adaptive OT using the assisted decryption approach. In priced OT, the obliviousness property must hold, even though the items being sold may have unique prices. The scheme is secure in the standard model under the Decision Linear, $q$-Triple Diffie-Hellman, and $q$-Hidden Strong Diffie-Hellman assumptions.

Unfortunately, all of these constructions have a rigid structure and seem to require a structured complexity assumption. We show that this structure can be enforced, not on the message itself, but rather through the identity of the encryption and the state of the signature. This provides us with enough glue to keep the security of the scheme together without overdoing it.

Recently, Kurosawa and Nojima 29] and Chen, Chou and Hou 14 gave adaptive OT constructions which purported to improve the underlying complexity assumptions of the schemes above, but which actually resorted to $O(N \lambda)$ transfer cost. It was already known how to achieve this level of (in)efficiency from general assumptions, including those of [2914], by following the folklore method for building adaptive OT from any non-adaptive OT system, as described in [1710 and the opening of our introduction. Moreover, 14 is set in the random oracle model.

Very recently2, Kurosawa, Nojima and Phong [30] gave an adaptive OT construction from DDH with $O(\lambda)$ transfers. However, their work has several technical issues. First, their construction does not satisfy the standard full simulation definition used in [10 21/22 27/36] and this work. In [30, if a receiver ever requests the same file twice (say, she downloads a patent one day, deletes it, then

$\overline{2}$ The work of 30 appeared after the initial posting of this work [23]. 
downloads it again a month later), then this can be detected by the sender. This is at odds with the full simulation definition where the adversarial sender is only told by the ideal functionality that a file has been requested and thus cannot detect a repeated download. Second, it is not obvious how to modify their construction to satisfy the full simulation notion. One approach might be to make the receiver stateful and store every file she ever requests. This has the obvious drawback of requiring permanent storage of the decrypted messages, which may not be practical and is not a requirement in other works. Moreover, subtle technical issues arise as to what the receiver sends during a repeated query round. Third, their construction requires a very expensive initialization procedure where the sender must transmit, then receive back and store $O(N \lambda)$ bits for each receiver. In contrast, all prior practical work 102122 27 36 and our results only require that the sender publish and store one $O(N \lambda)$ bit database for all receivers.

Thus, we build on this body of prior work to present the first efficient scheme satisfying the standard notion of full simulation from a simple assumption in the standard model.

\section{Technical Preliminaries}

Bilinear Groups. Let BMsetup be an algorithm that, on input $1^{\kappa}$, outputs the parameters for a bilinear mapping as $\gamma=\left(p, g, \mathbb{G}, \mathbb{G}_{T}, e\right)$, where $g$ generates $\mathbb{G}$, the groups $\mathbb{G}, \mathbb{G}_{T}$ have prime order $p \in \Theta\left(2^{\kappa}\right)$, and $e: \mathbb{G} \times \mathbb{G} \rightarrow \mathbb{G}_{T}$. Two algebraic properties required are that: (1) if $g$ generates $\mathbb{G}$, then $e(g, g) \neq 1$ and (2) for all $a, b \in \mathbb{Z}_{p}$, it holds that $e\left(g^{a}, g^{b}\right)=e(g, g)^{a b}$.

Assumption 1 (Decisional 3-Party Diffie-Hellman (3DDH) [31,5,25]) Let $\mathbb{G}$ be a group of prime order $p \in \Theta\left(2^{\lambda}\right)$. For all p.p.t. adversaries $\mathcal{A}$, the following probability is $1 / 2$ plus an amount negligible in $\lambda$ :

$$
\operatorname{Pr}\left[g, z_{0} \leftarrow \mathbb{G} ; a, b, c \leftarrow \mathbb{Z}_{p} ; z_{1} \leftarrow g^{a b c} ; d \leftarrow\{0,1\} ; d^{\prime} \leftarrow \mathcal{A}\left(g, g^{a}, g^{b}, g^{c}, z_{d}\right): d=d^{\prime}\right] .
$$

Proofs of Knowledge. We use known zero-knowledge and witness indistinguishable techniques for proving statements about discrete logarithms and their natural extensions to proving statements about bilinear groups, such as (1) proof of knowledge of a discrete logarithm modulo a prime [37] and (2) proof of the disjunction or conjunction of any two statements [16]. These are typically interactive, 4-round protocols. We discuss further implementation details in the full version 23 .

When referring to the proofs above, we will use the notation of Camenisch and Stadler [1]. For instance, $Z K \operatorname{KPoK}\left\{(x, h): y=g^{x} \wedge H=e(y, h) \wedge(1 \leq x \leq n)\right\}$ denotes a zero-knowledge proof of knowledge of an integer $x$ and a group element $h \in \mathbb{G}$ such that $y=g^{x}$ and $H=e(y, h)$ holds and $1 \leq x \leq n$. All values not enclosed in ()'s are assumed to be known to the verifier. 


\subsection{The Construction}

Our $\mathrm{OT}_{k \times 1}^{N}$ protocol appears in Figure 2 This protocol follows the assisted (or blind) decryption paradigm pioneered by [10|22]27]. The Sender begins the OT protocol by encrypting each message in the database and publishing these values to the Receiver. The Receiver then checks that each ciphertext is well-formed. For each of $k$ transfers, the two parties co-operatively execute a protocol following which (1) the Receiver obtains the decryption of at most one ciphertext, while (2) the Sender learns nothing about which ciphertext was decrypted. We require that the interactive decryption protocol run in time independent of the size of the database.

The encryption scheme that we use is a novel combination of the Boneh-Boyen IBE scheme [2] and a (insecure) version of the Hohenberger-Waters signatures [26]. We present methods for proving knowledge of such signatures and obtaining a blind decryption. Of course, in an adaptive OT scheme, the difficulty is always in getting all elements of the fully-simulatable proof of security to work out. There are many subtle details in basing the security for any database of size $N$ under the one simple assumption that given $\left(g, g^{a}, g^{b}, g^{c}\right)$, it is hard to decide if $Q=g^{a b c}$.

Ciphertext Structure. In Figure 2, we reference a VerifyCiphertext algorithm for verifying the well-formedness of a ciphertext. Let us explain that now. The Sender's public parameters $p k$ include $\gamma=\left(p, g, \mathbb{G}, \mathbb{G}_{T}, e\right)$ and generators $\left(g_{1}, g_{2}\right.$, $\left.h, g_{3}, g_{4}, u, v, d\right) \in \mathbb{G}^{8}$. For message $M \in \mathbb{G}_{T}$, identity $j \in \mathbb{Z}_{p}$, and random values $r, s, t \in \mathbb{Z}_{p}$ we can express a ciphertext as:

$$
C=\left(g^{r},\left(g_{1}^{j} h\right)^{r}, M \cdot e\left(g_{1}, g_{2}\right)^{r}, g^{t},\left(u^{r} v^{s} d\right)^{b}\left(g_{3}^{j} h\right)^{t}, u^{r}, s\right)
$$

Given only $p k, j$, the VerifyCiphertext function validates that the ciphertext has this structure. We define it as follows.

VerifyCiphertext $(p k, C, j)$. Parse $C$ as $\left(c_{1}, \ldots, c_{7}\right)$ and $p k$ to obtain $g, g_{1}, h, g_{3}, g_{4}$, $u, v, d$. This routine outputs 1 if and only if the following equalities hold:

$$
\begin{aligned}
e\left(g_{1}^{j} h, c_{1}\right) & =e\left(g, c_{2}\right) \wedge \\
e\left(g, c_{6}\right) & =e\left(c_{1}, u\right) \wedge \\
e\left(g, c_{5}\right) & =e\left(g_{4}, c_{6} v^{c_{7}} d\right) e\left(c_{4}, g_{3}^{j} h\right)
\end{aligned}
$$

This function will always output 1 when input a properly-formed ciphertext.

\subsection{Security Analysis}

We now show that the $\mathrm{OT}_{k \times 1}^{N}$ protocol above is sender-secure and receiversecure in the full-simulation model under the Decisional 3-Party Diffie-Hellman assumption (3DDH). We will address Sender and Receiver security separately.

$A$ note on the PoK protocols. For generality, our security proofs use the terms $\epsilon_{Z K}, \epsilon_{W I}$ to indicate the maximal advantage that every p.p.t. distinguisher has in distinguishing simulated ZKPoKs from real ones (resp. WI proofs on different witnesses). We additionally use $\epsilon_{E x t}$ to indicate the maximum probability that 


\section{$\underline{\mathrm{S}_{\mathrm{I}}\left(M_{1}, \ldots, M_{N}\right)} \quad \underline{\mathrm{R}_{\mathrm{I}}()}$}

1. Select $\gamma=\left(p, g, \mathbb{G}, \mathbb{G}_{T}, e\right) \leftarrow \operatorname{BMsetup}\left(1^{\kappa}\right)$ and $a, b \stackrel{\$}{\leftarrow} \mathbb{Z}_{p}$, choose $g_{2}, g_{3}, h, u, v, d \stackrel{\$}{\leftarrow} \mathbb{G}$ and set $g_{1} \leftarrow g^{a}, g_{4} \leftarrow g^{b}$.

Let $p k=\left(\gamma, g_{1}, g_{2}, g_{3}, g_{4}, h, u, v, d\right)$ and $s k=(a, b)$.

2. For $j=1$ to $N$, select $r_{j}, s_{j}, t_{j} \stackrel{\$}{\leftarrow} \mathbb{Z}_{p}$ and set:

$C_{j} \leftarrow\left[g^{r_{j}},\left(g_{1}^{j} h\right)^{r_{j}}, M_{j} e\left(g_{1}, g_{2}\right)^{r_{j}}, g^{t_{j}},\left(u^{r_{j}} v^{s_{j}} d\right)^{b}\left(g_{3}^{j} h\right)^{t_{j}}, u^{r_{j}}, s_{j}\right]$.

3. Send $\left(p k, C_{1}, \ldots, C_{N}\right)$ to Receiver.

4. Conduct $Z K \operatorname{PoK}\left\{(a): g_{1}=g^{a}\right\}$.

5. Verify $p k$ and the proof ${ }^{a}$.

Check for $j=1$ to $N$ :

VerifyCiphertext $\left(p k, C_{j}, j\right)=1$.

If any check fails, output $\perp$.

Output $S_{0}=(p k, s k)$.

Output $R_{0}=\left(p k, C_{1}, \ldots, C_{N}\right)$.

$\underline{\mathrm{S}_{\mathrm{T}}\left(S_{i-1}\right)}$

$\underline{\mathrm{R}_{\mathrm{T}}\left(R_{i-1}, \sigma_{i}\right)}$

1. Parse $C_{\sigma_{i}}$ as $\left(c_{1}, \ldots, c_{7}\right)$, select $x, y \stackrel{\$}{\leftarrow} \mathbb{Z}_{p}$ and compute $v_{1} \leftarrow g^{x} c_{1}$.

2. Send $v_{1}$ to Sender, and conduct:

$W I P o K\left\{\left(\sigma_{i}, x, c_{2}, c_{4}, c_{5}, c_{6}, c_{7}\right)\right.$ : $e\left(v_{1} / g^{x},\left(g_{1}^{\sigma_{i}} h\right)\right)=e\left(c_{2}, g\right) \wedge$ $e\left(c_{6}, g\right)=e\left(v_{1} / g^{x}, u\right) \wedge$

3. Set $R \leftarrow e\left(v_{1}, g_{2}^{a}\right)$. $\left.e\left(c_{5}, g\right)=e\left(c_{6} v^{c_{7}} d, g_{4}\right) e\left(c_{4}, g_{3}^{\sigma_{i}} h\right)\right\}$.

4. Send $R$ to Receiver and conduct: $Z K \operatorname{PoK}\left\{(a): R=e\left(v_{1}, g_{2}^{a}\right) \wedge g_{1}=g^{a}\right\}$.

5. If the proof does not verify, output $\perp$.

Else output $M_{\sigma_{i}}^{\prime} \leftarrow \frac{c_{3} \cdot e\left(g_{1}, g_{2}\right)^{x}}{R}$.

Output $S_{i}=S_{i-1} . \quad$ Output $R_{i}=\left(R_{i-1}, M_{\sigma_{i}}^{\prime}\right)$.

${ }^{a}$ By verify $p k$, we mean check that $\gamma$ contains parameters for a bilinear map, where $p$ is prime and $g$ generates $\mathbb{G}$ with order $p$. Also, verify that the remaining $p k$ elements are members of $\mathbb{G}$.

Fig. 2. Our adaptive $\mathrm{OT}_{k \times 1}^{N}$ protocol. VerifyCiphertext is described above.

the extractor for a PoK fails (soundness). We propose to use four-round Schnorr proofs which are zero-knowledge/WI $\left(\epsilon_{W I}=\epsilon_{Z K}=0\right)$ and computationally sound under the Discrete Logarithm assumption (which is naturally implied by 3DDH). Our security proofs employ the knowledge extractors for these proofsof-knowledge, which we will define as $E_{1}, E_{2}, E_{3} 3$.

3 These correspond respectively to the proofs $Z K \operatorname{PoK}\left\{(a): g_{1}=g^{a}\right\}$, $W I P o K\left\{\left(\sigma_{i}, x, y, z, c_{4}, c_{5}, c_{6}, c_{7}\right): \ldots\right\}$, and $Z K \operatorname{PoK}\left\{(a): R=e\left(v_{1}, g_{2}^{a}\right) \wedge g_{1}=g^{a}\right\}$. 
SENDER SECURITY. Given a (possibly cheating) real-world receiver $\hat{R}$, we show how to construct an ideal-world receiver $\hat{R}^{\prime}$ such that all p.p.t. distinguishers have at most negligible advantage in distinguishing the distribution of an honest real-world sender $S$ interacting with $\hat{R}\left(\mathbf{R e a l}_{\mathrm{S}, \hat{\mathrm{R}}}\right)$ from that of $\hat{\mathrm{R}}^{\prime}$ interacting with the honest ideal-world sender $\mathrm{S}^{\prime}\left(\mathbf{I d e a l}_{\mathrm{S}^{\prime}, \hat{\mathrm{R}}^{\prime}}\right)$. Let us now describe the operation of $\hat{\mathrm{R}}^{\prime}$, which runs $\hat{\mathrm{R}}$ internally, interacting with it in the role of the Sender:

1. To begin, $\hat{\mathrm{R}}^{\prime}$ selects a random collection of messages $\bar{M}_{1}, \ldots, \bar{M}_{N} \stackrel{\$}{\longleftarrow} \mathbb{G}_{T}$ and follows the $S_{I}$ algorithm (from Figure 2) with these as input up to the point where it obtains $\left(p k, C_{1}, \ldots, C_{N}\right)$.

2. It sends $\left(p k, C_{1}, \ldots, C_{N}\right)$ to $\hat{\mathrm{R}}$ and then simulates the interactive proof $Z K \operatorname{PoK}\left\{(a): g_{1}=g^{a}\right\}$. (Even though $\hat{\mathrm{R}}^{\prime}$ knows $s k=a$, it ignores this value and simulate this proof step.)

3. For each of $k$ transfers initiated by $\hat{\mathrm{R}}$,

(a) $\hat{\mathrm{R}}^{\prime}$ verifies the received WIPoK and uses the knowledge extractor $\mathrm{E}_{2}$ to obtain the values $\sigma_{i}, x, y, c_{1}, c_{2}, c_{3}, c_{4}$ from it. $\hat{\mathrm{R}}^{\prime}$ aborts and outputs error when $\mathrm{E}_{2}$ fails.

(b) When $\sigma_{i} \in[1, N], \hat{\mathrm{R}}^{\prime}$ queries the trusted party $T$ to obtain $M_{\sigma_{i}}$, parses $C_{\sigma_{i}}$ as $\left(c_{1}, \ldots, c_{7}\right)$ and responds with $R=\frac{c_{3} e\left(g_{1}, g_{2}\right)^{x}}{M_{\sigma_{i}}}$ (if $T$ returns $\perp, \hat{\mathrm{R}}^{\prime}$ aborts the transfer). When $\sigma_{i} \notin[1, N], \hat{\mathrm{R}}^{\prime}$ follows the normal protocol. In both cases, $\hat{\mathrm{R}}^{\prime}$ simulates $Z K \operatorname{PoK}\left\{(a): R=e\left(v_{1}, g_{2}^{a}\right) \wedge g_{1}=g^{a}\right\}$.

4. $\hat{R}^{\prime}$ uses $\hat{R}$ 's output as its own.

Theorem 2. Let $\epsilon_{Z K}$ be the maximum advantage with which any p.p.t. algorithm distinguishes a simulated $Z K P o K$, and $\epsilon_{E x t}$ be the maximum probability that the extractor $\mathrm{E}_{2}$ fails (with $\epsilon_{Z K}$ and $\epsilon_{E x t}$ both negligible in $\kappa$ ). If all p.p.t. algorithms have negligible advantage $\leq \epsilon$ at solving the $3 D D H$ problem, then:

$$
\begin{gathered}
\operatorname{Pr}\left[D\left(\mathbf{R e a l}_{\mathrm{S}, \hat{\mathrm{R}}}\left(N, k, M_{1}, \ldots, M_{N}, \Sigma\right)\right)=1\right]- \\
\operatorname{Pr}\left[D\left(\mathbf{I d e a l}_{\mathrm{S}^{\prime}, \hat{\mathrm{R}}^{\prime}}\left(N, k, M_{1}, \ldots, M_{N}, \Sigma\right)\right)=1\right] \leq \\
(k+1) \epsilon_{Z K}+k \epsilon_{E x t}+N \epsilon\left(1+\frac{p}{p-1}\right) .
\end{gathered}
$$

A proof of Theorem 2 is sketched in Appendix A.1 and detailed in [23].

RECEIVER SECURITY. For any real-world cheating sender $\hat{S}$ we can construct an ideal-world sender $\hat{S}^{\prime}$ such that all p.p.t. distinguishers have negligible advantage at distinguishing the distribution of the real and ideal experiments. Let us now describe the operation of $\hat{S}^{\prime}$, which runs $\hat{S}$ internally, interacting with it in the role of the Receiver.

1. To begin, $\hat{S}^{\prime}$ runs the $R_{\mathrm{I}}$ algorithm, with the following modification: when $\hat{\mathrm{S}}$ proves knowledge of $a, \hat{\mathrm{S}}^{\prime}$ uses the knowledge extractor $\mathrm{E}_{1}$ to extract $a$, outputting error if the extractor fails. Otherwise, it has obtained the values $\left(p k, C_{1}, \ldots, C_{N}\right)$. 
2. For $i=1$ to $N, \hat{\mathrm{S}}^{\prime}$ decrypts each of $\hat{\mathrm{S}}$ 's ciphertexts $C_{1}, \ldots, C_{N}$ using the value $a$ as a decryption key 4 and sends the resulting $M_{1}^{*}, \ldots, M_{N}^{*}$ to the trusted party $T$.

3. Whenever $T$ indicates to $\hat{S}^{\prime}$ that a transfer has been initiated, $\hat{S}^{\prime}$ runs the transfer protocol with $\hat{S}$ on the fixed index 1. If the transfer succeeds, $\hat{S}^{\prime}$ returns the bit 1 (indicating success) to $T$, or 0 otherwise.

4. $\hat{\mathrm{S}}^{\prime}$ uses $\hat{\mathrm{S}}^{\prime}$ s output as its own.

Theorem 3. Let $\epsilon_{W I}$ be the maximum advantage that any p.p.t. algorithm has at distinguishing a WIPoK, and let $\epsilon_{\text {Ext }}$ be the maximum probability that the extractor $\mathrm{E}_{1}$ fails. Then $\forall$ p.p.t. $D$ :

$$
\begin{aligned}
\operatorname{Pr} & {\left[D\left(\boldsymbol{R e a l}_{\hat{\mathrm{S}}, \mathrm{R}}\left(N, k, M_{1}, \ldots, M_{N}, \Sigma\right)\right)=1\right]-} \\
& \operatorname{Pr}\left[D\left(\operatorname{Ideal}_{\hat{\mathrm{S}}^{\prime}, \mathrm{R}^{\prime}}\left(N, k, M_{1}, \ldots, M_{N}, \Sigma\right)\right)=1\right] \leq(k+1) \epsilon_{E x t}+k \epsilon_{W I} .
\end{aligned}
$$

A proof of Theorem 3 is sketched in Appendix A.2 and detailed in [23.

\section{Efficiently Supporting Multiple Receivers}

Adaptive Oblivious Transfer $\left(\mathrm{OT}_{k \times 1}^{N}\right)$ is traditionally defined as a protocol between a Sender and a single Receiver. However, the way it is typically used in practical works such as Coull et al. [15] and Camenisch et al. [8] is that $U \geq 1$ distinct Receivers all interact with a single Sender.

Extending the full simulation definition to cover this explicitly is rather straightforward. We do so in the full version [23]. The main technical concern is that every Receiver should have the same view of the database. That is, if two Receivers make a request on index $i$ and neither transfer resulted in an error, then those Receivers must have obtained the same message. In [23] we explain why our construction would satisfy such a notion - namely, that all Receivers share the same database and a Receiver does not accept a message unless the Sender can prove that it correctly corresponds to this database. For simplicity, we assume secure channels for the transfer phase.

Eliminating the $O(\lambda U)$ term. Interestingly, we can also improve the efficiency of our initialization protocol when multiple Receivers are present. In the current protocol of Figure 2, the Sender must conduct the proof of knowledge $Z K \operatorname{PoK}\left\{(a): g_{1}=g^{a}\right\}$ with each Receiver. This can be accomplished using a very inexpensive interactive four-round proof in the standard model.

Fortunately even this minimal per-user initialization can be eliminated by assuming a Common Reference String shared by the Sender and all Receivers and using an NIZKPoK to broadcast this proof to all Receivers. To instantiate this proof, we suggest the efficient proof system of Groth and Sahai [24,

${ }^{4}$ Parse $C_{i}$ as $\left(c_{1}, \ldots, c_{7}\right)$ and decrypt as $M_{i}^{*}=c_{3} / e\left(c_{1}, g_{2}^{a}\right)$. As noted in Section 3 , one can modify the protocol so that the Sender conducts a PoK of the value $g_{2}^{a}$. 
which permits proofs of pairing-based statements under the Decision Linear assumption [4]. One wrinkle in this approach is that our proof of Receiver security assumes that the simulator can extract the trapdoor $a \in \mathbb{Z}_{p}$ from the ZKPoK, in order to decrypt the ciphertext vector $C_{1}, \ldots, C_{N}$. However, the knowledge extractor for the Groth-Sahai proof system is limited in that it can only extract elements of the bilinear image group $\mathbb{G}$. Fortunately for our purposes, the value $g_{2}^{a} \in \mathbb{G}$ can be used as an alternative trapdoor (see Section 2.2 for details). Thus when using the Groth-Sahai system we must re-write the proof as $N I Z K \operatorname{PoK}\left\{\left(g_{2}^{a}\right): e\left(g_{1}, g_{2}\right)=e\left(g_{2}^{a}, g\right)\right\}$.

\section{Conclusions and Open Problems}

We presented the first efficient, adaptive oblivious transfer protocol which is fully-simulatable under simple, static assumptions. Our protocol is practical and can be used as a building block in larger database applications, such as [1536], as a step to reducing the overall assumptions on the system.

We leave open several interesting problems. First, we use standard zeroknowledge proof and extraction techniques which require rewinding, and thus, our scheme is not UC-secure. A natural question is whether one can obtain UC-security by replacing our interactive proofs with the non-interactive GrothSahai proofs 24. Unfortunately, this is not an easy substitution. Our security proofs use techniques from the Boneh-Boyen cryptosystem that depend fundamentally on the ability to extract integers from the Receiver's proof of knowledge during the Transfer phase. The Groth-Sahai proof system is only $F$-extractable, meaning that one can obtain only group elements from the extractor (even when the proof is over integer witnesses). One can easily substitute a bit-by-bit proof of each integer, but we would hope to identify a more practical approach.

It would be interesting to know if the observations about and manipulations of the Hohenberger-Waters signatures [26] identified in this work could be extended to applications such as anonymous credentials and electronic cash, where most efficient constructions still require random oracles or strong complexity assumptions. One of the main difficulties is that many interesting protocols require an $F$-signature together with an efficient range proof (i.e., method for proving in zero-knowledge that a committed value lies within a public range.) Currently, the only efficient techniques for doing the latter require either the Strong RSA assumption [13 96] or (more recently) the $q$-Strong Diffie-Hellman assumption [7]12]. (Here $q$ need only be linked to a security parameter, e.g., $q=256$.) It would be interesting if range proofs under weaker assumptions could be devised.

\footnotetext{
${ }^{5}$ As mentioned by Groth and Sahai, statements of this form must be slightly rewritten to enable full zero knowledge. The equivalent statement is $Z K \operatorname{PoK}\left\{\left(g_{2}^{a}, g_{1}^{\prime}\right)\right.$ : $\left.e\left(g_{2}^{a}, g\right) e\left(g_{1}^{\prime}, g_{2}^{-1}\right)=1 \wedge e\left(g_{1}^{\prime}, g\right)=e\left(g_{1}, g\right)\right\}$.
} 


\section{References}

1. Belenkiy, M., Chase, M., Kohlweiss, M., Lysyanskaya, A.: P-signatures and Noninteractive Anonymous Credentials. In: Canetti, R. (ed.) TCC 2008. LNCS, vol. 4948, pp. 356-374. Springer, Heidelberg (2008)

2. Boneh, D., Boyen, X.: Efficient Selective-ID Secure Identity-Based Encryption Without Random Oracles. In: Cachin, C., Camenisch, J.L. (eds.) EUROCRYPT 2004. LNCS, vol. 3027, pp. 223-238. Springer, Heidelberg (2004)

3. Boneh, D., Boyen, X.: Short Signatures Without Random Oracles. In: Cachin, C., Camenisch, J.L. (eds.) EUROCRYPT 2004. LNCS, vol. 3027, pp. 56-73. Springer, Heidelberg (2004)

4. Boneh, D., Boyen, X., Shacham, H.: Short Group Signatures. In: Franklin, M. (ed.) CRYPTO 2004. LNCS, vol. 3152, pp. 41-55. Springer, Heidelberg (2004)

5. Boneh, D., Sahai, A., Waters, B.: Fully Collusion Resistant Traitor Tracing with Short Ciphertexts and Private Keys. In: Vaudenay, S. (ed.) EUROCRYPT 2006. LNCS, vol. 4004, pp. 573-592. Springer, Heidelberg (2006)

6. Boudot, F.: Efficient Proofs that a Committed Number Lies in an Interval. In: Preneel, B. (ed.) EUROCRYPT 2000. LNCS, vol. 1807, pp. 431-444. Springer, Heidelberg (2000)

7. Camenisch, J., Chaabouni, R., Shelat, A.: Efficient Protocols for Set Membership and Range Proofs. In: Pieprzyk, J. (ed.) ASIACRYPT 2008. LNCS, vol. 5350, pp. 234-252. Springer, Heidelberg (2008)

8. Camenisch, J., Dubovitskaya, M., Neven, G.: Oblivious transfer with access controls. In: ACM CCS 2009, pp. 131-140 (2009)

9. Camenisch, J., Michels, M.: Proving in Zero-Knowledge that a Number $n$ is the Product of Two Safe Primes. In: Stern, J. (ed.) EUROCRYPT 1999. LNCS, vol. 1592, pp. 107-122. Springer, Heidelberg (1999)

10. Camenisch, J., Neven, G., Shelat, A.: Simulatable Adaptive Oblivious Transfer. In: Naor, M. (ed.) EUROCRYPT 2007. LNCS, vol. 4515, pp. 573-590. Springer, Heidelberg (2007)

11. Camenisch, J., Stadler, M.: Efficient Group Signature Schemes for Large Groups. In: Kaliski Jr., B.S. (ed.) CRYPTO 1997. LNCS, vol. 1294, pp. 410-424. Springer, Heidelberg (1997)

12. R. Chaabouni, H. Lipmaa, A. Shelat. Additive combinatorics and discrete logarithm based range protocols (2009), Cryptology ePrint Archive: 2009/469 http://eprint.iacr.org/2009/469.pdf

13. Chan, A., Frankel, Y., Tsiounis, Y.: Easy Come - Easy Go Divisible Cash. In: Nyberg, K. (ed.) EUROCRYPT 1998. LNCS, vol. 1403, pp. 561-575. Springer, Heidelberg (1998)

14. Chen, Y., Chou, J.-S., Hou, X.-W.: A novel k-out-of-n oblivious transfer protocols based on bilinear pairings (2010), Cryptology ePrint Archive: Report 2010/027

15. Coull, S., Green, M., Hohenberger, S.: Controlling Access to an Oblivious Database Using Stateful Anonymous Credentials. In: Jarecki, S., Tsudik, G. (eds.) PKC 2009. LNCS, vol. 5443, pp. 501-520. Springer, Heidelberg (2009)

16. Cramer, R., Damgård, I., Schoenmakers, B.: Proof of Partial Knowledge and Simplified Design of Witness Hiding Protocols. In: Desmedt, Y.G. (ed.) CRYPTO 1994. LNCS, vol. 839, pp. 174-187. Springer, Heidelberg (1994)

17. Dodis, Y., Halevi, S., Rabin, T.: A Cryptographic Solution to a Game Theoretic Problem. In: Bellare, M. (ed.) CRYPTO 2000. LNCS, vol. 1880, pp. 112-130. Springer, Heidelberg (2000) 
18. Dodis, Y., Yampolskiy, A.: A Verifiable Random Function with Short Proofs and Keys. In: Vaudenay, S. (ed.) PKC 2005. LNCS, vol. 3386, pp. 416-431. Springer, Heidelberg (2005)

19. Goldreich, O., Micali, S., Wigderson, A.: How to play any mental game or a completeness theorem for protocols with honest majority. In: STOC 1987, pp. 218-229 (1987)

20. Goldwasser, S., Micali, S., Rivest, R.L.: A digital signature scheme secure against adaptive chosen-message attacks. SIAM J. Computing 17(2) (1988)

21. Green, M., Hohenberger, S.: Blind Identity-Based Encryption and Simulatable Oblivious Transfer. In: Kurosawa, K. (ed.) ASIACRYPT 2007. LNCS, vol. 4833, pp. 265-282. Springer, Heidelberg (2007)

22. Green, M., Hohenberger, S.: Universally Composable Adaptive Oblivious Transfer. In: Pieprzyk, J. (ed.) ASIACRYPT 2008. LNCS, vol. 5350, pp. 179-197. Springer, Heidelberg (2008)

23. Green, M., Hohenberger, S.: Practical adaptive oblivious transfer from simple assumptions (2010), The full version of this work appears in the Cryptology ePrint Archive: Report 2010/109

24. Groth, J., Sahai, A.: Efficient Non-interactive Proof Systems for Bilinear Groups. In: Smart, N.P. (ed.) EUROCRYPT 2008. LNCS, vol. 4965, pp. 415-432. Springer, Heidelberg (2008)

25. Hohenberger, S., Rothblum, G.N., Shelat, A., Vaikuntanathan, V.: Securely Obfuscating Re-encryption. In: Vadhan, S.P. (ed.) TCC 2007. LNCS, vol. 4392, pp. 233-252. Springer, Heidelberg (2007)

26. Hohenberger, S., Waters, B.: Realizing Hash-and-Sign Signatures under Standard Assumptions. In: Joux, A. (ed.) EUROCRYPT 2009. LNCS, vol. 5479, pp. 333-350. Springer, Heidelberg (2009)

27. Jarecki, S., Liu, X.: Efficient Oblivious Pseudorandom Function with Applications to Adaptive OT and Secure Computation of Set Intersection. In: Reingold, O. (ed.) TCC 2009. LNCS, vol. 5444, pp. 577-594. Springer, Heidelberg (2009)

28. Kilian, J.: Founding cryptography on oblivious transfer. In: STOC 1988, pp. 20-31 (1988)

29. Kurosawa, K., Nojima, R.: Simple Adaptive Oblivious Transfer without Random Oracle. In: Matsui, M. (ed.) ASIACRYPT 2009. LNCS, vol. 5912, pp. 334-346. Springer, Heidelberg (2009)

30. Kurosawa, K., Nojima, R., Phong, L.T.: Efficiency-Improved Fully Simulatable Adaptive OT under the DDH Assumption. In: Garay, J.A., De Prisco, R. (eds.) SCN 2010. LNCS, vol. 6280, pp. 172-181. Springer, Heidelberg (2010)

31. Laguillaumie, F., Paillier, P., Vergnaud, D.: Universally Convertible Directed Signatures. In: Roy, B. (ed.) ASIACRYPT 2005. LNCS, vol. 3788, pp. 682-701. Springer, Heidelberg (2005)

32. Lindell, Y.: Efficient Fully-Simulatable Oblivious Transfer. In: Malkin, T.G. (ed.) CT-RSA 2008. LNCS, vol. 4964, pp. 52-70. Springer, Heidelberg (2008)

33. Naor, M., Pinkas, B.: Oblivious Transfer with Adaptive Queries. In: Wiener, M. (ed.) CRYPTO 1999. LNCS, vol. 1666, pp. 573-590. Springer, Heidelberg (1999)

34. Peikert, C., Vaikuntanathan, V., Waters, B.: A Framework for Efficient and Composable Oblivious Transfer. In: Wagner, D. (ed.) CRYPTO 2008. LNCS, vol. 5157, pp. 554-571. Springer, Heidelberg (2008)

35. Rabin, M.: How to exchange secrets by oblivious transfer. Technical Report TR-81, Aiken Computation Laboratory, Harvard University (1981) 
36. Rial, A., Kohlweiss, M., Preneel, B.: Universally Composable Adaptive Priced Oblivious Transfer. In: Shacham, H., Waters, B. (eds.) Pairing 2009. LNCS, vol. 5671, pp. 231-247. Springer, Heidelberg (2009)

37. Schnorr, C.-P.: Efficient signature generation for smart cards. Journal of Cryptology 4(3), 239-252 (1991)

38. Sun, H.-M., Chen, Y., Chou, J.-S.: An efficient secure oblivious transfer (2009), Cryptology ePrint Archive: Report 2009/521

39. Wiesner, S.: Conjugate coding. SIGACT News 15, 78-88 (1983)

40. Yao, A.: How to generate and exchange secrets. In: FOCS, pp. 162-167 (1986)

\section{A Proof Sketches of Sender and Receiver Security}

Complete proofs of sender and receiver security appear in the full version [23].

\section{A.1 Proof Sketch of Sender Security (Theorem 2)}

Proof. We will begin with $\mathbf{R e a l}_{\mathrm{S}, \hat{\mathrm{R}}}$, then modify the distribution via a series of hybrid games until we arrive at a distribution identical to that of $\mathbf{I d e a l}_{\mathrm{S}^{\prime}, \hat{\mathrm{R}}^{\prime}}$. Let us define these hybrids as follows:

Game 0. The real-world experiment conducted between $S$ and $\hat{R}\left(\mathbf{R e a l}_{\mathrm{S}, \hat{\mathrm{R}}}\right)$. Game 1. This game modifies Game 0 as follows: (1) each of S's ZKPoK executions is replaced with a simulated proof of the same statement 6 and (2) the knowledge extractor $\mathrm{E}_{2}$ is used to obtain the values $\left(\sigma_{i}, x, y, z, \bar{c}_{4}, \bar{c}_{5}, \bar{c}_{6}, \bar{c}_{7}\right)$ from each of $\hat{\mathrm{R}}$ 's transfer queries. Whenever the extractor fails, $\mathrm{S}$ terminates the experiment and outputs the distinguished symbol error.

Game 2. This game modifies Game 1 such that, whenever the extracted value $\sigma_{i} \in[1, N]$, S's response $R$ is computed using the following approach: parse $C_{\sigma_{i}}=\left(c_{1}, \ldots, c_{7}\right)$ and set $R=\frac{c_{3} e\left(g_{1}, g_{2}\right)^{x}}{M_{\sigma_{i}}}$. When $\sigma_{i} \notin[1, N]$, the response is computed using the normal protocol.

Game 3. This game modifies Game 2 by replacing the input to $S_{\text {I }}$ with a dummy vector of random messages $\bar{M}_{1}, \ldots, \bar{M}_{N} \in \mathbb{G}_{T}$. However when S computes a response value using the technique of Game 2, the response is based on the original message vector $M_{1}, \ldots, M_{N}$. We claim that the distribution of this game is equivalent to that of $\mathbf{I d e a l}_{\mathrm{S}^{\prime}, \hat{\mathrm{R}}^{\prime}}$.

Let us now consider the following Lemmas. For notational convenience, define:

$\operatorname{Adv}[$ Game $\mathbf{i}]=\operatorname{Pr}[D($ Game $\mathbf{i})=1]-\operatorname{Pr}[D($ Game 0$)=1]$.

Lemma 1. If all p.p.t. algorithms $D$ distinguish a simulated ZKPoK with advantage at most $\epsilon_{Z K}$ and the extractor $\mathrm{E}_{2}$ fails with probability at most $\epsilon_{E x t}$, then $\mathbf{A d v}\left[\right.$ Game 1] $\leq(k+1) \cdot \epsilon_{Z K}+k \cdot \epsilon_{E x t}$.

${ }^{6}$ This includes at most $k+1$ PoK executions, including the initial $Z K \operatorname{PoK}\left\{(a): g_{1}=\right.$ $\left.g^{a}\right\}$ and each subsequent proof $Z K \operatorname{PoK}\left\{(a): R=e\left(v_{1}, g_{2}^{a}\right) \wedge g_{1}=g^{a}\right\}$. 
Lemma 2. If no p.p.t. algorithm has advantage $>\epsilon$ in solving the $3 D D H$ problem, then

$$
\mathbf{A d v}[\text { Game 2 }]-\mathbf{A d v}\left[\text { Game 1] } \leq \frac{N p}{p-1} \cdot \epsilon .\right.
$$

Lemma 3. If no p.p.t adversary has advantage $>\epsilon$ at solving the 3DDH problem, then

$$
\operatorname{Adv}[\text { Game 3] }-\mathbf{A d v}[\text { Game 2] } \leq N \cdot \epsilon .
$$

Proof of the above lemmas is in 23]. By summing over hybrids Game 0 to Game 3, we obtain Adv [Game 3] $\leq(k+1) \epsilon_{Z K}+k \epsilon_{E x t}+N \epsilon\left(1+\frac{p}{p-1}\right)$. For the Schnorr proofs we use, $\epsilon_{Z K}=0$. This concludes the proof of Sender security.

\section{A.2 Proof Sketch of Receiver Security (Theorem 3)}

Proof. We again arrive at the ideal-world sender via a series of hybrid games:

Game 0. The real-world experiment conducted between $\hat{S}$ and $R\left(\mathbf{R e a l}_{\hat{\mathrm{S}}, \mathrm{R}}\right)$. Game 1. A modification of Game $\mathbf{0}$ in which $\mathrm{R}$ applies the knowledge extractor $\mathrm{E}_{1}$ to $\hat{\mathrm{S}}^{\prime}$ s proof $\mathrm{ZKPoK}\left\{a: g_{1}=g^{a}\right\}$. If this extraction fails, R aborts and outputs $\perp$. Further, for transfers $i=1$ through $k, \mathrm{R}$ uses the knowledge extractor $\mathrm{E}_{3}$ on $\hat{\mathrm{S}}$ 's proof $Z K \operatorname{PoK}\left\{(a): R=e\left(v_{1}, g_{2}^{a}\right) \wedge g_{1}=g^{a}\right\}$ to extract the values $a$, aborting if the extractor fails (or returns inconsistent values). Game 2. For transfer $i=1$ to $k$, modify R's request such that $\sigma_{i}=1$. The distribution of this game is identical to that of $\mathbf{I d e a l}_{\hat{\mathrm{S}}^{\prime}, R^{\prime}}$.

Lemma 4. If the extractor $\mathrm{E}_{1}$ and $\mathrm{E}_{3}$ fail with probability at most $\epsilon_{E x t}$, then $\operatorname{Adv}\left[\right.$ Game 1] $\leq(k+1) \epsilon_{E x t}$.

Lemma 5. If the Receiver's WIPoK is distinguishable with maximum advantage $\epsilon_{W I}$, then

$$
\operatorname{Adv}[\text { Game 2 }]-\mathbf{A d v}\left[\text { Game 1] }=\leq k \cdot \epsilon_{W I} .\right.
$$

Proof of the above lemmas is in 23 . Summing the differences, we have

$$
\mathbf{A d v}[\text { Game 2 }]-\mathbf{A d v}[\text { Game 0 }]=(k+1) \epsilon_{E x t}+k \epsilon_{W I} .
$$

For the Schnorr proofs we use, $\epsilon_{W I}=0$. This concludes the proof of Receiver security. 